\title{
NLOS Identification Based on Energy Receiver Using IEEE 802.15.3c
}

\author{
Meng Zhang ${ }^{1,}$, Hao Zhang $^{1,2, b}$ and Xiaolin Liang ${ }^{1, c}$ \\ ${ }^{1}$ College of Information Science and Engineering, Ocean University of China, \\ Qingdao, 266100, China \\ ${ }^{2}$ Department of Electrical and Computer Engineering, University of Victoria \\ Victoria, V8W 3P6, Canada \\ azhangmeng7526@126.com, 'bhanghao@ouc.edu.cn, ‘xiaolin87liang@163.com
}

Keywords: NLOS identification, slope, skewness, $60 \mathrm{GHz}$, Energy Detection.

\begin{abstract}
Non-line-of-sight (NLOS) is the major problem for the indoor localization. So as to deal with NLOS propagation, a novel NLOS identification method is proposed based on the skewness and slope of the (energy detection) ED-based received energy block. IEEE 802.15.3c $60 \mathrm{GHz}$ channel models are used as examples to be explained in detail. The proposed approach relies on the ED-based parameters which make it simple. Numerical simulations results show that the accuracy of NLOS identification up to $80 \%$ which is higher than other ED-based NLOS identification.
\end{abstract}

\section{Introduction}

Advances in the $60 \mathrm{GHz}$ millimeter wave, the wireless communications in the $60 \mathrm{GHz}$ frequency band have attracted various attentions from both academia and industry for the past few years. According to the Federal Communications Commission, the unlicensed frequency spectrum between 57 and $64 \mathrm{GHz}$ for commercial use is freed, offering up to $7 \mathrm{GHz}$ in bandwidth [1]. Consequently, the various complementary metal oxide semiconductor devices are expected to achieve several Gbps data rates and will be able to support the bandwidth intensive, upcoming applications such as High-Definition Television. Moreover, the $60 \mathrm{GHz}$ is a key enabler of future, gigabits wireless networks, which encompasses next generation cellular communications [2] and body area networks [3].

The propagation range of millimeter wave radio signals, and in particular signals at frequencies around $60 \mathrm{GHz}$, is much more limited than that of radio signals at lower frequencies, as the higher frequency signals are significantly affected by the presence of oxygen and water vapor within the atmosphere. The location of a mobile terminal can be estimated based on different parameters of a received signal, such as the time of arrival, angle of arrival, and/or the received signal strength. Impulse radio-60GHz has a great potential for the accurate ranging and localization systems due to its very wide bandwidth and capability in resolving individual multipath components [4-6]. Therefore, the time of arrival of the received signal can be estimated with high accuracy for the $60 \mathrm{GHz}$ systems if the first arriving path has been identified precisely. One of the major challenges for localization systems is the mitigation of non-line of sight effects. If the direct path between a fixed terminal and the mobile terminal is being obstructed, the time of arrival of the signal to the fixed terminal will be delayed, which introduces a positive bias. Using such non-line of sight time of arrival estimates during the localization of the mobile terminal position may significantly degrade the positioning accuracy. Hence, the fixed terminals that are under the non-line of sight condition have to be identified and their effects have to be mitigated.

The non-line of sight identification techniques have been discussed extensively in the literature, but mainly within the cellular network framework [7-13]. For example, in [10], the authors address the NLOS identification problems based on the multiple received signal strength measurements from Wi-Fi signals. Shimizu et al. [12] performed intensive measurements of path-loss and delay-profile characteristics of line-of-sight and non-line-of-sight environments in a suburban residential area. Based on their analysis, they found that the delay spread was dependent on distance, and the 
non-line-of-sight delay spread was found to be several times larger than that of the line-of-sight case. The skewness of delay spread for the non-line-of-sight cases ranged from 80 to 200ns, which was an order of magnitude larger than that of the line-of-sight case.

In this paper, a new NLOS identification approach is proposed for the impulse radio $60 \mathrm{GHz}$ signal, which is based on the slope and skewness of energy block of the received signal based on ED. Firstly, we use the energy detect time of arrival estimation algorithm for the estimation the TOA. Secondly, we characterize the slope and skewness of the energy block of the received signal. Finally, we use a threshold test for the NLOS identification. The remainder of this paper is organized as follows. Section 2 describes the signal and channel model. Section 3 describes NLOS identification approach and section 4 presents the results of the numerical simulations. The concluding remarks are given in section 5 .

\section{System Model}

Currently, there are two important standards that have been developed for the $60 \mathrm{GHz}$ wireless communications systems, IEEE 802.15.3c and IEEE 802.11ad [14]. In this paper, the channel models in IEEE 802.15. 3c standard are used because it is specifically designed for the wireless personal area networks and thus encompasses typical indoor environments. IEEE 802.15.3c standard was the first developed for high data rate short-range wireless systems. The physical layer was designed to support the transmission of data within a few meters at a maximum data rate of 2 Gbps. These models have been developed for communications in the frequency band 57 to $66 \mathrm{GHz}$ in indoor residential, indoor office and library environments [15-16]. The transmitted $60 \mathrm{GHz}$ signals can be expressed as:

$$
s(t)=\sum_{-\infty}^{\infty} p\left(t-j T_{s}-C_{j} T_{c}-a_{j} \varepsilon\right)
$$

where $T_{s}$ is the symbol time. The time hopping code represented by $C_{j}$ is a pseudorandom integer-valued sequence which is unique for each user to limit multiple access interference, and $T_{c}$ is the chip time. The pulse position modulation time shift is $\varepsilon$ so that if $a_{j}$ is 1 , the signal is shifted in time by $\varepsilon$, while if $a_{j}$ is 0 , there is no shift. Many pulse shapes have been proposed for the $60 \mathrm{GHz}$ systems. In this paper a Gaussian pulse is employed which is multiplied by the carrier signal to give:

$$
p(t)=\frac{\sqrt{2}}{\alpha} \exp \left(-2 \pi \frac{t^{2}}{\alpha^{2}}\right) \cos (2 \pi f t)
$$

where $\alpha$ is the shape factor and $f$ is the carrier frequency which here is $f=60.5 \mathrm{GHz}$. A smaller shape factor results in a shorter duration pulse and a larger bandwidth. The received signal can be written as:

$$
r(t)=\sum_{n=1}^{N} \alpha_{n} p\left(t-\tau_{n}\right)+n(t)=r(t)=s(t) * h(t)+n(t)
$$

where $N$ is the number of received multipath components, $\alpha_{n}$ and $\tau_{n}$ denotes the amplitude and delay of the $n$th path respectively, $n(t)$ is the additive white Gaussian noise with zero mean and two sided power spectral density $\mathrm{N}_{0} / 2$, and $h(t)$ is the channel response which can be expressed as:

$$
h(t, \theta)=\sum_{k=1}^{K} \sum_{l=1}^{L_{k}} \mu_{k l} \delta\left(t-T_{k}-\tau_{k l}\right) \delta\left(\theta-\theta_{k}-\omega_{k l}\right)
$$

where $\delta($.$) is the dirac delta function, K$ is the number of clusters, $L_{k}$ is the number of rays in the $k^{\text {th }}$ cluster, and $\mu_{k l}, \tau_{k l}$ and $\omega_{k l}$ denote the complex amplitude, delay and azimuth of the $k^{\text {th }}$ ray of the $l^{\text {th }}$ cluster, respectively. Similarly, $T_{k}$ and $\theta_{k}$ represent the delay and mean AOA of the $k^{\text {th }}$ cluster. As shown in Fig.1, after the amplifier, the received signals are squared, and then input to an integrator with integration period $T b$. The integrator outputs can be expressed as:

$$
z[n]=\sum_{i=1}^{N} \int_{(i-1) T_{s}+\left(c_{j}+n-1\right) T b}^{(i-1) T_{T}+\left(c_{j}+n\right) T b} r^{2}(t) d t
$$




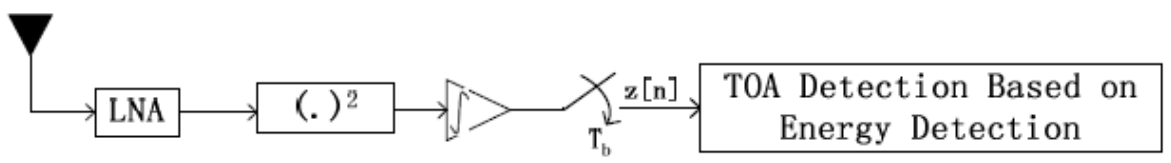

Fig. 1. Block diagram of the energy detection receiver

where $n \in\{1,2, . ., N\}$ denotes the sample index with respect to the starting point of the integration period and $N$ is the number of pulses per symbol. Here, $N$ is set to 1 , so the integrator outputs are:

$$
z[n]=\sum_{i=1}^{N} \int_{\left(c_{j}+n-1\right) T b}^{\left(c_{j}+n\right) T b} r^{2}(t) d t
$$

If $z[n]$ is the integration of noise only, it has a centralized Chi-square distribution, while it has a non-centralized Chi-square distribution if a signal is present.

\section{NLOS Identification}

In this paper, we distinguish NLOS scenarios by exploiting the statistics of the received signal by ED. The slope and skewness of the energy block is used in order to identify NLOS scenarios respectively. The slope of the energy values is considered as a measure. These values are divided into (N-M+1) groups, with $\mathrm{M}$ values in each group. The slope for each group is calculated using a least squares line-fit. The maximum slope can then be expressed as:

$$
M S=\underset{1 \leq n \leq N-M+1}{\operatorname{slope}}\{\text { linefit }(z[n], z[n+1], \ldots . ., z[n+M-1])\}
$$

The skewness is given by:

$$
S=\frac{1}{(N-1) \delta^{3}} \sum_{i=1}^{N}\left(x_{i}-\mu_{x}\right)^{3}
$$

where $\mu_{x}$ is the mean value, and $\delta$ is the standard deviation of the energy values. The skewness for a normal distribution is 0 , in fact any symmetric data will have a skewness of zero. Negative values of skewness indicate that the data is skewed left, while positive values indicate data that is skewed right. Skewed left indicates that the left tail is long relative to the right tail, while skewed right indicates the opposite. For noise only (or very low SNR), $S=0$. As the SNR increases, $S$ will tend to increase.

The ratio values of the slope and skewness of the energy block can be obtained for NLOS scenarios using sample. Here, we used sample channel realizations of the IEEE 802.15.3c channel models to obtain the ratio values for LOS and NLOS. In order to examine the characteristics of the ratio, the CM1.1 and CM2.1 are employed. For each SNR value, 1000 channel realizations are generated and sampled at $f=10 \mathrm{GHz}$. The other system parameters are $T_{s}=200 n s, T_{c}=1 n s, T b$ is 1 ns and 4ns, and $N=1$. Each realization has a TOA uniformly distributed within $\left(0-T_{s}\right)$. Here the SNR ranges from 0 to $9 \mathrm{~dB}$. The residential LOS and NLOS channel models from IEEE 802.15.3c standard are employed. Here are $10 * 1000$ samples which are got for each channel model. The relationship between the ratio and SNR are shown in Figs. 2-3. Results show the ratio is monotonous with respect to SNR in $60 \mathrm{GHz}$ system. But the minimum of the ratio in LOS is larger than the maximum in NLOS when $T X$ is $360^{\circ}$, the maximum in LOS is less than the minimum in NLOS when $T X$ is others. So we propose a novel method to identify the NLOS which can be expressed as:

$$
\Theta=\alpha\left\{\begin{array}{l}
\left\{\begin{array}{l}
>\alpha_{M S} \Rightarrow \text { LOS } \\
<\alpha_{M S} \Rightarrow N L O S
\end{array} \quad \text { TX }=360^{\circ}\right. \\
\left\{\begin{array}{l}
>\alpha_{M S} \Rightarrow N L O S \\
<\alpha_{M S} \Rightarrow \text { LOS }
\end{array} \quad\right. \text { others }
\end{array}\right.
$$

where $\alpha_{M S}$ is the threshold which is chosen to identify NLOS. TX, $R X$ is the beam-width of measured transmitter and receiver antenna respectively. It can be shown as: 


$$
\alpha_{M S}=\min \left\{\begin{array}{l}
\text { mean }\left[\text { find }\left(P\left((\text { Joint Metric })<\left.\Theta_{1}\right|_{A}\right) \geq \Phi\right)\right] \\
\text { mean }\left[\text { find }\left(P\left((\text { Joint Metric })<\left.\Theta_{2}\right|_{B}\right) \geq \Phi\right)\right]
\end{array}\right\}
$$

where $\Theta_{1}$ and $\Theta_{2}$ are threshold where SNR is upper and lower limits. $\Phi$ is the probability value which is required for choosing suitable threshold for NLOS identification. Without loss of generality, here $\Theta$ is 0.85 .

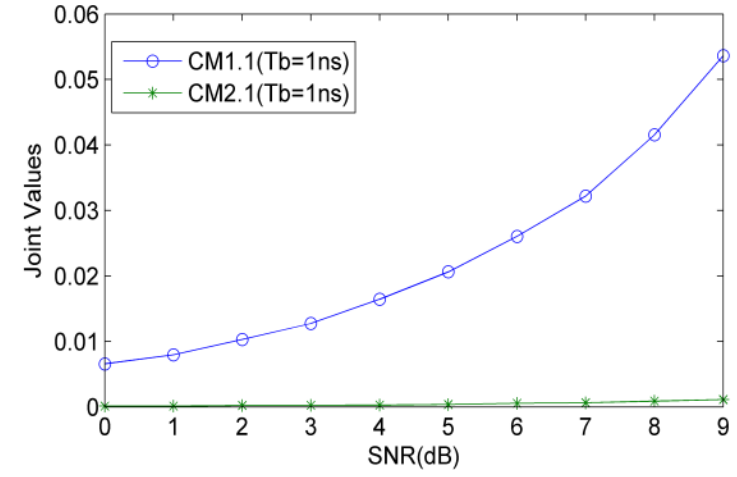

(1) $T b=1 \mathrm{~ns}$

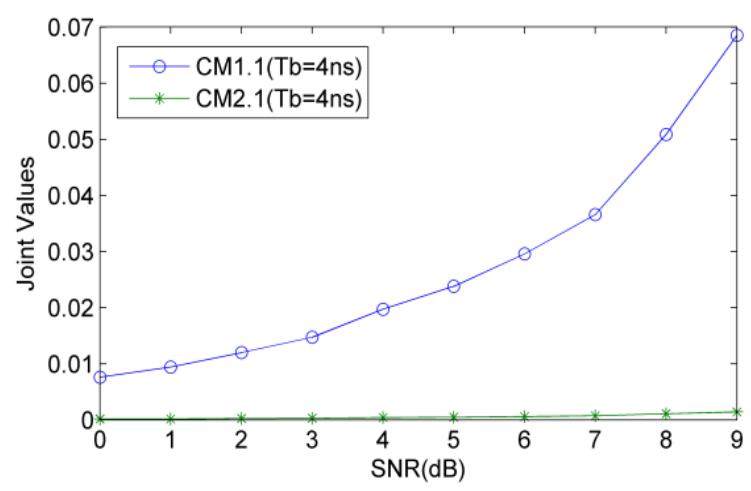

(2) $T b=4 \mathrm{~ns}$

Fig. 2. Joint Values with Respect to SNR $\left(\mathrm{TX}=360^{\circ}\right)$

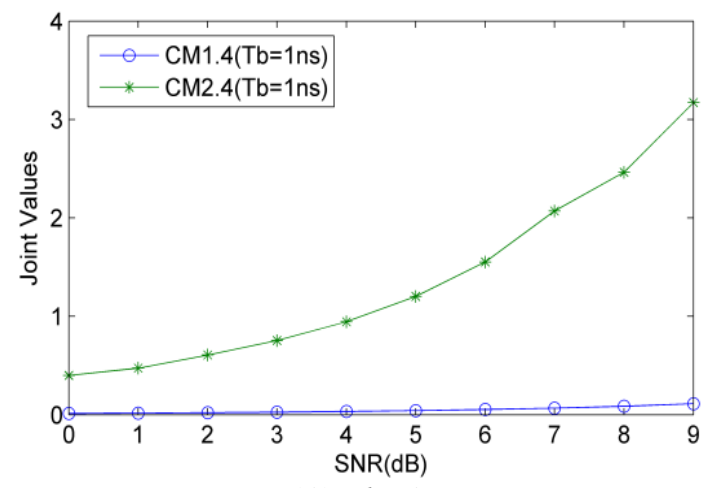

(1) $T b=1 \mathrm{~ns}$

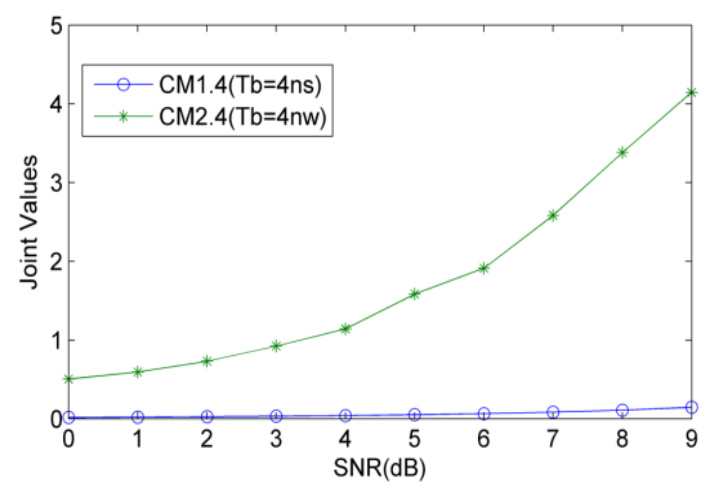

(2) $T b=4 \mathrm{~ns}$

Fig. 3. Joint Values with Respect to SNR $\left(\mathrm{TX}<360^{\circ}\right)$

In order to verify the effectiveness and practicality of the algorithm, so we make a lot of simulations using IEEE 802.15.3c channel models. The CM1.2, CM1.3, CM1.4 (residential LOS) and CM2.2, CM2.3, CM2.4 (residential NLOS) channel models from IEEE802.15.3c are employed. SNR ranges from 0 to $9 \mathrm{~dB}, 1000$ channel realizations are generated for each SNR. Here 10*1000 samples are got for each channel model. The relationship between joint parameter and SNR are shown in Figs. 2-3. Results show the accuracy of NLOS identification is higher than any other identification algorithms based on ED such 60\% while the accuracy of the proposed algorithm over $80 \%$ for the most channel model.

\section{Conclusion}

In this paper, we presented a novel approach to deal with non-line-of-sight propagation that relies solely on features extracted from the received waveform. This technique does not require formulation of explicit statistical models for the features which is based on the slope and skewness of energy block of the received signal using ED. In order to verify the effectiveness and practicality of the algorithm, so we make a lot of simulations using IEEE 802.15.3c channel models. The residential LOS and residential NLOS channel models from the IEEE 802.15.3c standard are employed. Results show that the joint parameter can identify the LOS and NLOS environments so long as the threshold can be fixed bitterly. We developed techniques that are capable of distinguishing LOS/NLOS propagation in NLOS conditions. Our results revealed that the proposed technique outperforms previous parametric techniques from the literature. 


\section{Acknowledgments}

This work was supported by the Nature Science Foundation of China under Grant No. 60902005, the Qingdao International Science and Technology Cooperation Projects of Qingdao under Grant No. 12-1-4-137-hz, and the Qingdao Transformation of Scientific and Technological Achievements Guiding Plan under Grant No. 14-2-4-37-jch.

\section{Reference}

[1] R.C. Daniels, J.N. Murdock, T.S. Rappaport and R.W. Heath: IEEE Microw. Mag. Vol. 11 (2010), p. 44.

[2] Z. Pi and F. Khan: IEEE Commun. Mag. Vol. 49 (2011), p. 101.

[3] N. Chahat, G. Valerio, M. Zhadobov and R. Sauleau: IEEE Trans. Antennas Propagat. Vol. 61 (2013), p. 1876.

[4] L. Zhang: Commun. China, Vol. 11 (2014), p. 42.

[5] W. Zhang, Y. P. Zhang, M. Sun, C. Luxey, D. Titz and F. Ferrero: IEEE T Antenn Propag, Vol. 61 (2013), p. 6228.

[6] D. Grujic, M. Savic, C. Bingöl and L. Saranovac: IEEE Micro. Wirel. Components Lett., Vol. 22 (2012), p. 194.

[7] K. Yu and E, Dutkiewicz: IEEE Trans Aerosp Electron Syst, Vol. 49 (2013), p. 1438.

[8] S. Tian, L. Zhao and G. Li: Math Probl Eng, Vol. 2014 (2014), doi.10.1155/2014/963418.

[9] Z. Wang, S. Zekavat and A. Reza: IEEE T Mobile Comput, Vol. 11 (2012), p. 2047-2059.

[10] Z. Xiao, H. Wen, A. Markham, N. Trigoni, P. Blunsom and J. Frolik: IEEE Tran Wirel Commun, Vol. 14 (2015), p. 1689.

[11] S. Wu, J. Li and S. Liu: Int J Commun Syst, Vol. 27 (2014), p. 2156.

[12] S. Venkatesh and R. M. Buehrer: IET Microw Antenna P, Vol. 1 (2007), p.1120.

[13] I. Güvenç, C. C. Chong, F. Watanabe and H. Inamura: EURASIP J Adv Sig Pro, Vol. 2008 (2008).

[14] C. R. Andersonn and T. S. Rappaport: IEEE T Wirel Commun, Vol. 3 (2004), p. 922.

[15] S. Collong, G. Zaharia and G. E. Zein: IEEE T Wirel Commun, Vol. 3 (2005), p. 2396.

[16] A. Maltsev, R. Maslennikov and A. Sevastyanov: IEEE J Sel Area Comm, Vol. 27 (2009), p. 1488. 